\title{
Linx
}

Revue des linguistes de l'université Paris X Nanterre

$79 \mid 2019$

Enseigner et apprendre à interagir en langue étrangère : réflexions linguistiques et didactiques

\section{Matériel authentique et développement de la compréhension orale en interaction}

Authentic material and the development of oral comprehension in interaction

\section{Simone MOREHED et Anita THOMAS}

\section{OpenEdition}

\section{Journals}

Édition électronique

URL : http://journals.openedition.org/linx/3514

DOI : $10.4000 /$ linx.3514

ISSN : 2118-9692

Éditeur

Presses universitaires de Paris Nanterre

Référence électronique

Simone MOREHED et Anita THOMAS, « Matériel authentique et développement de la compréhension orale en interaction », Linx [En ligne], 79 | 2019, document 3, mis en ligne le 30 décembre 2019,

consulté le 10 décembre 2020. URL : http://journals.openedition.org/linx/3514 ; DOI : https://doi.org/ 10.4000/linx.3514

Ce document a été généré automatiquement le 10 décembre 2020.

Département de Sciences du langage, Université Paris Ouest 


\section{Matériel authentique et développement de la compréhension orale en interaction}

Authentic material and the development of oral comprehension in interaction

Simone MOREHED et Anita THOMAS

\section{Introduction}

1 La mise à disposition de corpus de recherche de français parlé est une ressource qui permet de développer l'enseignement de phénomènes linguistiques et interactionnels qui ne sont que peu ou mal représentés dans les manuels de langue étrangère ou seconde (dorénavant L2). Bien que l'interaction soit une activité centrale au niveau de l'utilisation d'une langue donnée, en particulier dans les orientations communicatives et agentives de l'apprentissage des L2, les propositions d'enseignement de celle-ci en classe de langue restent rares (Ravazzolo et al. 2015). Traditionnellement, la compréhension et la production orales sont enseignées et testées séparément, par exemple par le biais de jeux de rôles plus ou moins préfabriqués ou d'exercices de compréhension orale plus ou moins authentiques. Pourtant, l'interaction est une activité co-construite entre les interactante-s, dans laquelle production et compréhension s'enchaînent presque immédiatement. Les recherches sur le développement de la compétence d'interaction en L2 indiquent que les compétences interactionnelles acquises en L1 ne suffisent pas en L2, comme le montrent par exemple les études sur l'humour de Shively (2013). Les résultats de ces études suggèrent qu'il est nécessaire d'inclure les spécificités de l'interaction, par exemple la gestion des désaccords ou les méthodes d'atténuation (Kreutel 2007), dans l'enseignement d'une L2.

2 La plupart des recherches sur la compétence d'interaction en L2 se sont intéressées à la production. L'aspect compréhension de l'interaction a été peu exploré comme le signalent Culpeper, Mackey et Taguchi dans le domaine de la pragmatique : 
Hearers have been neglected in traditional pragmatics research, which has a speaker bias [...]. Hearers are particularly important in L2 pragmatics, where listening, as well as speaking, is essential in our understanding of how a second language is learned. (Culpeper, Mackey et Taguchi $2018: 90$ )

Dans cette contribution, nous nous intéresserons à la compréhension de séquences de désaccord et d'accord en interaction par des apprenant·e's L2, et en particulier à la manière dont les corpus d'interaction disponibles en ligne peuvent servir de ressource pour l'enseignement et l'apprentissage de la compréhension dans le cadre de l'interaction. Un des avantages de l'utilisation des corpus comme ressource didactique est que ceux-ci donnent accès à une utilisation authentique de la langue, à savoir des interactions enregistrées dans le contexte où elles ont été produites par les interactant·es de manière spontanée, à la différence de matériel spécialement construit pour l'enseignement (Boulton \& Tyne 2014).

4 Nous nous focaliserons sur ce que les apprenante's peuvent retenir de cette sorte de matériel. En fait, nous ne savons pas encore en détail ce que comprennent les apprenante-s de différents niveaux d'une interaction authentique ni ce qui leur pose vraiment problème. L'objectif de cet article est donc de discuter de la manière dont on peut étudier la compréhension dans le cadre d'une interaction.

\section{Les dimensions linguistiques, interactionnelles et pragmatiques de la compréhension orale}

5 Savoir interagir dans une L2 est primordial, l'interaction étant l'outil par lequel nous accomplissons la plupart des activités de la vie quotidienne (Gülich \& Mondada 2001). Cette compétence comprend des compétences linguistiques ainsi que des compétences pragmatiques: non seulement il faut comprendre ce qui est dit aux niveaux linguistiques et sémantiques, mais également aux niveaux contextuels et culturels (Rost $2016: 67)$.

6 La compréhension a été étudiée dans différents contextes et avec différentes méthodes. On trouve d'une part l'approche traditionnelle de la compréhension orale, où cette dernière est étudiée de manière plus ou moins isolée du contexte interactif (Bloomfield et al. 2010) et d'autre part l'approche interactionnelle, dans laquelle on évalue principalement la compréhension à partir de ce qui est produit plus tard dans la conversation (Oursel 2013; Skogmyr, Petitjean et Pekarek Doehler 2017; Traverso 2017). Finalement, les études en pragmatique L2 s'intéressent, quant à elles, à la compréhension (et à la production subséquente) de différentes activités interactionnelles ou d'implicatures conversationnelles (Culpeper, Mackey et Taguchi 2018). Ces différentes approches de la compréhension seront présentées dans ce qui suit.

\subsection{La compréhension orale}

7 Comprendre un message oral est complexe et requiert différents processus: neurologiques (p. ex. la réception et la conversion des ondes sonores), linguistiques ( $\mathrm{p}$. ex. la perception et le traitement de la langue), sémantiques (la construction de sens à partir de différents indices), et pragmatiques (la compréhension du sens communiqué par l'interactante, à partir d'indices contextuels) (Rost 2016). À partir de la vaste revue 
de la littérature sur la compréhension orale en L2 de Bloomfield et al. (2010), on constate que les aspects interactionnels sont largement absents dans les études sur la compréhension orale; les activités de compréhension orale servent plutôt à vérifier le niveau de connaissances linguistiques (de la grammaire et du vocabulaire), traditionnellement évaluées en L2 que les connaissances pragmatiques et contextuelles. De plus, dans les études et les tâches de compréhension orale traditionnelle, on a souvent recours à un matériel didactique et construit. Les caractéristiques propres à l'interaction authentique, par exemple des répétitions, hésitations, et chevauchements font de ce fait défaut dans ce type de matériel. Des études comparant le matériel authentique et le matériel construit ont montré que le matériel authentique comprend plus de répétitions et plus de mots, mais moins de densité lexicale et de complexité syntaxique que le matériel construit (Bloomfield et al. $2010: 42$; Gilmore 2004 et 2007).

Un autre point important concernant le matériel authentique est qu'il n'est pas créé pour convenir à un niveau spécifique de langue des apprenante's; le matériel authentique peut ainsi contenir des formes, expressions, ou situations interactionnelles que les apprenantees ne connaissent pas ou auxquelles ils/elles ne sont pas habituée's.s. Les études antérieures sur le sujet indiquent que la compréhension orale est rendue plus difficile si le message contient des mots et expressions culturellement spécifiques à la L2 (par exemple des expressions idiomatiques), car ces mots et expressions risquent d'être mal interprétés (Bloomfield et al. 2010).

Outre le type de matériel utilisé, les conditions dans lesquelles le niveau de compréhension des apprenant·e's est évalué sont également d'une grande importance, en particulier le temps à disposition pour compléter la tâche et le nombre d'écoutes d'un enregistrement. Les rares études dans ce domaine indiquent qu'une limite de temps pour finir la tâche la rend plus difficile. En même temps, la pression du temps est intrinsèque à la compréhension orale vu que le processus d'écoute est immédiat et en ligne (Bloomfield et al. 2010: 70). Ceci est une des caractéristiques de l'interaction authentique, où le message communiqué doit être compris sous la contrainte du débit de l'émetteur, un facteur qui touche aussi au nombre d'écoutes d'un enregistrement. Les études ayant testé ce facteur ont montré que les apprenant·es se rappelaient plus de choses après une deuxième écoute qu'après une seule (Bloomfield et al $2010: 72$ ). Comme le signale Oursel (2013: 505), la possibilité d'écoute répétée est une caractéristique de l'activité de compréhension orale en classe de langue. Selon elle, une seule écoute serait à préférer afin que les apprenante-s puissent apprendre à écouter dans des conditions similaires à un contexte d'interaction et à gérer le stress d'une seule écoute.

\subsection{Interaction et intercompréhension}

10 La compréhension a été indirectement étudiée dans les recherches sur la compétence d'interaction en L2 par le biais de l'étude des mécanismes de co-construction et donc de la production des interactant·es. Ces études se sont intéressées à différentes activités interactionnelles telles que le désaccord (Pochon-Berger \& Pekarek Doehler 2011, Fasel Lauzon et al. 2009, Kreutel 2007, Bardovi-Harlig \& Salsbury 2004) ou l'humour (Skogmyr et al. 2017, Shively 2013). Le but de ces études est d'en savoir plus sur le développement des compétences qui permettent à l'apprenante d'interagir dans la L2 d'une manière 
adéquate et donc de repérer les procédures interactionnelles qui contribuent au développement d'une L2.

11 A partir de ces études nous savons que la manière de s'exprimer et d'accomplir des activités interactionnelles se développe et se complexifie avec le temps. Dans leur étude de l'humour en français L2, Skogmyr et al. (2017) ont analysé des interactions au sein de deux groupes d'apprenant·ess du français L2 (niveaux A2 et B2) et ont trouvé que les deux groupes réussissaient à utiliser différentes ressources interactionnelles dans le but de communiquer de l'humour en interaction. Les apprenante's avancée's utilisaient des ressources plus complexes que les apprenant·es débutant·es, qui, eux/elles, avaient recours à des ressources plus simples, comme le rire. Shively (2013) par contre, dans son étude longitudinale d'un apprenant américain de l'espagnol L2, montre comment les échecs répétés de l'apprenant le mènent à changer de forme d'humour en développant des stratégies comme des signaux prosodiques, lexicaux et morphosyntaxiques dans le but de contextualiser ses blagues, ainsi qu'en imitant différents dialectes. Selon Shively (2013), ces résultats suggèrent que les compétences pragmatiques peuvent se développer par la participation aux interactions, même si ces compétences se développent parfois lentement en L2 et ne convergent pas forcément vers ce que font les natif've's.

Dans sa thèse de doctorat, Oursel (2013) s'est plus particulièrement penchée sur les processus d'intercompréhension, qu'elle définit comme étant le "résultat d'un processus collaboratif d'élaboration du sens par les interactants» (Oursel 2013: 51), c'est-à-dire " une interprétation commune des énoncés échangés » (Oursel 2013:4). Il s'agit donc d'étudier les ressources interactionnelles communiquant des difficultés de compréhension ou d'autres ruptures de la communication. Le niveau de compréhension est de ce fait analysé à partir de la production, souvent dans des paires adjacentes dans lesquelles on observe par exemple une réponse inappropriée ou l'introduction d'une séquence de négociation de sens.

Plus précisément, Oursel (2013) a étudié des interactions de services administratifs en français entre agent·e (natif.ve) et client·e (non-natif·ve) dans le but de voir comment des négociations et explications de différents types sont utilisées par les interactant-e's afin d'arriver à une intercompréhension. Selon elle, les interactante-s interprètent les énoncés des interlocuteurstrices en se basant sur des connaissances supposées "partagées"; des connaissances qui ne sont pas les mêmes entre les individus et les cultures (Oursel 2013: 36). On voit ainsi que le développement de la compétence d'interaction ne dépend pas seulement du développement linguistique, mais aussi du développement des connaissances culturelles et contextuelles de l'interaction.

\subsection{La compétence pragmatique en L2}

14 La compréhension a également été étudiée dans le domaine de la pragmatique L2 (Taguchi 2015). Ce sont surtout des expressions ou activités pragmatiques spécifiques, par exemple l'ironie ou la requête, ou des expressions implicites comme l'implicature, qui ont été étudiées de manière expérimentale (cf. Culpeper, Mackey et Taguchi 2018; Bardovi-Harlig 2009 sur les expressions conventionnelles en anglais L2; Shively et al. 2008 sur la compréhension de l'ironie en espagnol L2; Taguchi 2005 sur la compréhension de l'implicature conversationnelle en anglais L2). Les méthodes employées sont entre autres le discourse completion test (DCT), où l'apprenant·e doit 
compléter la suite d'une interaction à l'écrit ou à l'oral, le jeu de rôle, les questions à choix multiples, ou encore les questions de jugements où il/elle doit juger si un énoncé est approprié ou non selon le contexte interactionnel donné (Culpeper, Mackey et Taguchi 2018 : 90ss). Ici, on peut constater que le matériel utilisé comme stimulus est fréquemment un matériel construit, bien que les situations décrites soient censées illustrer une interaction orale authentique. De plus, ces tests se font souvent à l'écrit, même si un input audio et multimodal commence à être utilisé (cf. Taguchi, GomezLaich et Arrufat-Marques 2016 ; Bardovi-Harlig 2009).

Les résultats de ces études montrent que la compréhension en pragmatique en L2 varie selon les niveaux de L2 des apprenantee's, ainsi qu'entre les apprenante's et les interactant-e's natif've's. Taguchi, Gomez-Laich et Arrufat-Marques (2016), par exemple, ont étudié la compréhension de trois types d'activités indirectes dans des interactions en espagnol L2: le refus indirect, l'expression indirecte d'une opinion, et l'ironie. Les données d'input correspondaient à des enregistrements vidéo construits à partir de situations authentiques. Chaque vidéo était visualisée aux apprenant·es qui ensuite répondaient à des questions à choix multiples avec 4 alternatives comprenant des affirmations sur le contenu de la conversation, dont une seule était correcte. Taguchi et al. (2016) ont trouvé une différence significative entre les apprenante's et le groupe de contrôle de interactant-e's natif-ve's d'espagnol, quant aux réponses aux questions ainsi qu'au temps de réponse, les natif·ve's répondant plus souvent correctement, et plus rapidement que les apprenantees. Les auteurs concluent sur la nécessité d'étudier la compréhension du sens implicite, vu la difficulté qu'ont les apprenantees L2 à le comprendre.

16 Au sujet du matériel construit utilisé dans leur étude, Taguchi et al. (2016) signalent qu'elles ont fait des modifications à partir du matériel original authentique pour qu'il ne soit pas trop difficile à comprendre par les apprenante's de niveau intermédiaire. Les dialogues présentés dans l'étude n'étaient donc pas proprement authentiques mais plutôt inspirés par des dialogues authentiques. Taguchi et al. (2016) suggèrent d'inclure à l'avenir des apprenante's avancée's pour pouvoir ainsi « explore creative ways to incorporate truly naturalistic spoken discourse into the assessment of indirectness comprehension " (Taguchi et al. 2016: 695). On peut se demander si un tel matériel ne pourrait pas aussi être utilisé avec des apprenant·e·s de niveaux moins avancés. C'est ce que nous avons étudié dans le cadre de deux études que nous présenterons et discuterons dans la suite de cet article.

\subsection{Conclusion des études antérieures sur la compréhension en L2}

Ce tour d'horizon d'études antérieures selon différentes approches, nous permet de noter la diversité des ressources utilisées pour l'étude de la compréhension. Les tâches de compréhension orale et les études pragmatiques se basent le plus souvent sur du matériel construit alors que les études sur l'interaction et l'intercompréhension se basent sur du matériel produit en ligne par les apprenantees eux/elles-mêmes. La question qui se pose ainsi est de savoir si l'on pourrait arriver à une situation intermédiaire, dans laquelle on utiliserait du matériel authentique mais de manière différée, dans le cadre d'un design de type expérimental.

Les corpus d'interactions authentiques pourraient ici constituer une vraie ressource en ce qui concerne la recherche et l'enseignement des aspects interactionnels et 
pragmatiques d'une langue donnée (Ravazzolo \& Etienne, ce volume). En effet, les enregistrements authentiques nous fournissent l'usage "réel» d'une langue, à la différence des explications et définitions qu'on trouve souvent dans les livres de grammaires et les manuels scolaires. De plus, les corpus permettent d'inclure les aspects contextuels qui ont une place centrale et importante en interaction, par exemple quant aux différentes manières de s'exprimer dans un contexte professionnel comparé à un contexte privé. La variation dans une langue, largement absente dans le matériel construit, peut également s'observer par les corpus (Debaisieux 2009).

\section{3. Étude hors ligne du volet 'compréhension' de l'interaction}

Pour illustrer comment le volet 'compréhension' en interaction peut être étudié de manière plus expérimentale et à partir d'interactions authentiques, nous présenterons et discuterons une étude sur la compréhension du désaccord en interaction en français qui a été menée en 2017 (Morehed 2017). Cette étude non publiée se base sur l'étude de Thomas et al. (2016) que nous discuterons également plus en détail. Les deux études s'appuient en effet sur le même matériel authentique sur la thématique du désaccord. Nous commençons donc par présenter une définition du désaccord et des études antérieures ayant traité la production du désaccord en L2.

\subsection{Le désaccord en interaction}

20 Le désaccord est un phénomène interactionnel central et fréquent en interaction (Kerbrat-Orecchioni 1992). On peut considérer le désaccord comme une activité interactionnelle paradoxale dans la mesure où un certain niveau de désaccord est requis en interaction, sans quoi les interactantees risquent de n'avoir rien à se dire, en même temps qu'un niveau élevé de désaccord peut menacer les relations interpersonnelles des interactante's et le déroulement même de l'interaction (Traverso 1996). Le désaccord est donc une activité interactionnelle importante que les apprenant·e·s, en particulier ceux de niveaux avancés, doivent apprendre à maîtriser de manière adéquate.

21 Le désaccord peut se définir comme étant une « manifestation discursive d'opposition à l'encontre du dire ou du faire d'autrui » (Pochon-Berger et Pekarek Doehler 2011 : 246), ou comme une "divergence d'opinion par rapport à un discours antérieur " (Fasel Lauzon et al. 2009 : 124). Voici un exemple (1) tiré du corpus CLAPI ${ }^{1}$ et discuté dans Thomas et al. (2016:23-24) :

\section{ANT c'est un peu long à cuire les magrets BRU mais c'est pas- c'est pas très long hein/}

Dans cet exemple, le premier interactant (ANT) exprime une affirmation, 'les magrets sont un peu long à cuire', avec laquelle le deuxième interactant (BRU) n'est pas d'accord (Ursi 2016). Cet énoncé contient l'idée contraire de celui de ANT, c'est-à-dire «le magret de canard est long à cuire " vs. "le magret de canard n'est pas long à cuire ». Les indices de désaccord se trouvent à la fois dans l'utilisation du marqueur 
d'opposition «mais » suivi d'une négation « c'est pas » répétée deux fois et un « hein » montant final qui sert d'adoucisseur.

Le désaccord en interaction est un phénomène qui a été examiné dans des études s'intéressant au développement de la compétence d'interaction L2. Ces études nous indiquent qu'il constitue un phénomène interactionnel complexe et pourtant important pour mener à bien une interaction.

Dans une étude sur la compétence d'interaction en français L2 d'élèves de niveau intermédiaire (secondaire I) et de niveau avancé (secondaire II), Pochon-Berger \& Pekarek Doehler (2011) ont trouvé que seulees les apprenante's plus avancéee's produisent le désaccord de manière non immédiate et non initiale et suivent donc le principe de la préférence pour l'accord ou du délai du désaccord mis en évidence par Pomerantz (1984). Les apprenantees de niveau intermédiaire expriment souvent le désaccord en position initiale, parfois avec un simple "non », ou avec la formulation mais $+X$. Cette étude montre que les apprennante's diversifient les moyens linguistiques et élargissent le "répertoire permettant d'exprimer un désaccord à proprement parler " (Pochon-Berger \& Pekarek Doehler 2011: 255). On trouve ainsi chez les apprenantees de niveau avancé l'utilisation du oui mais, le recyclage des structures ou encore l'utilisation de la question rhétorique, par exemple tu crois vraivraiment? (Pochon-Berger \& Pekarek Doehler 2011 : 254). Ces apprenante-s ne semblent donc pas avoir de peine à mettre en place les ressources interactionnelles de la L2 (voir aussi Mondada \& Pekarek Doehler 2000, 2005 ; Pekarek Doehler 2012).

Des résultats similaires ont été trouvés chez des apprenantees de l'anglais L2. Les stratégies utilisées dans la production d'un désaccord se développaient selon une trajectoire allant d'un désaccord fort, essentiellement "non ", à la diversification des stratégies qui ressemblent plus à la production des interactante-s L1 (Bardovi-Harlig et Salsbury 2004; Kreutel 2007). Les deux études signalent cependant certaines différences entre les apprenant·e's avancés et les interactant·e's L1. Les apprenant·e·s ont tendance à moins atténuer un désaccord, et à le faire seulement à la fin de l'énoncé, alors que les interactante's $\mathrm{L} 1$ le font et au début et à la fin de l'énoncé qui contient le désaccord (Kreutel 2007 : 4). Bardovi-Harlig et Salsbury (2004) ainsi que Kreutel (2007) concluent que les apprenantees ne maîtrisent pas les stratégies d'atténuation du désaccord, et qu'un enseignement sur ces aspects prépareraient mieux les apprenant·e's à ce type d'interaction. La conversation pourrait être utilisée en tant qu'outil pédagogique pour le développement de l'expression orale des apprenant-e's. BardoviHarlig et Salsbury (2004) affirment également l'importance des corpus oraux dans l'étude des aspects faisant partie de l'interaction, sans lesquels des points centraux, telle l'organisation des tours de paroles, ne peuvent pas être observés. On constate par contre que ces études se sont surtout intéressées au volet production de l'interaction. L'objectif des deux études que nous présenterons ci-après était donc de cibler la compréhension du désaccord en interaction.

\subsection{Compréhension d'extraits authentiques : questions posées oralement}

26 L'étude de Thomas et al. (2016), à laquelle l'étude de Morehed qui sera présentée plus en détail ci-dessous (voir 3.3) fait suite, avait pour objectif d'examiner la possibilité de relier des extraits du corpus d'interactions familières authentiques CLAPI à des niveaux 
du CECR (Cadre Européen Commun de Référence pour les langues) à partir du niveau de compréhension d'apprenante-s. En d'autres termes, il s'agissait donc de voir si le niveau de compréhension des apprenanteess variait selon le niveau de difficulté estimé pour chaque extrait.

Cette étude a été effectuée en deux étapes. Premièrement, les enregistrements choisis ont été analysés du point de vue interactionnel dans le but d'identifier les ressources et passages clés du désaccord présenté dans ces enregistrements. Par la suite, à partir des éléments relevés par cette analyse, on a déterminé le niveau de chaque enregistrement en les comparant aux descripteurs du CECR. Finalement un protocole expérimental a été construit dans le but d'étudier la relation entre le niveau de difficulté des extraits estimé à partir de l'analyse interactionnelle et la compréhension des éléments-clés par 22 apprenantee's du français de niveaux A2 à C1.

La méthode employée comprenait différentes questions posées oralement à l'apprenante individuellement après un ou plusieurs visionnements de l'extrait. L'approche était de type de non intervention et de non interprétation pour laisser le plus de place possible à l'apprenant·e à son interprétation et compréhension de l'extrait visionné. Les apprenant·e-s étaient encouragée's à répondre en français, mais pouvaient autrement le faire dans leur L1 (le suédois).

Les résultats ont indiqué que tous les enregistrements étaient accessibles aux apprenante's de tous les niveaux de français, mais que le niveau de détail et de précision de la compréhension pouvait varier selon les enregistrements. Plus les apprenant·es étaient avancée's, plus le niveau de compréhension des enregistrements, en particulier au niveau micro (détaillé) augmentait. Ils/elles ne comprenaient pas que le sujet et les grandes lignes du déroulement, mais pouvaient également expliquer le déroulement plus en détails. Les différences entre les niveaux de compréhension pouvaient s'expliquer par des contraintes liées aux niveaux lexical, contextuel et interactionnel, donc des caractéristiques inhérentes aux interactions choisies pour l'étude.

Du point de vue méthodologique, cette approche très ouverte a montré que la gestion de la compréhension se faisait de différentes manières selon les niveaux des apprenant·e's. Les plus débutant'e-s ont parfois eu de la peine à comprendre le sujet de l'extrait et sont partie's sur une mauvaise piste en raison d'une difficulté de compréhension de certains indices (linguistiques et paraverbaux) (Thomas et al. 2016 : 31). Bien que ce résultat soit intéressant, il révèle aussi les limites d'une méthode de verbalisation très ouverte pour étudier la compréhension, car elle ne permet pas de cibler suffisamment précisément ce qui pose problème au niveau de la compréhension en interaction.

\subsection{Compréhension d'extraits authentiques : questionnaire numérique}

31 Ainsi Morehed (2017) a mené une nouvelle étude dont le but était de tester la compréhension de manière plus directive et détaillée. Plus précisément, le but était de voir comment des apprenante's avancée's suédophones de français L2 comprennent le désaccord en conversation familière en français entre interactant·e's natif.ve's, surtout au niveau des différentes ressources interactionnelles produites dans des séquences de désaccord. Le but était également de faire une comparaison avec des interactant·ess 
natif.ve's du français afin de voir quelles sont les similarités et différences entre ces deux groupes.

\subsubsection{Démarche méthodologique} interactionnelle plus approfondie des deux séquences de désaccord utilisées dans Thomas et al. (2016). Les deux extraits ont été analysés afin d'extraire les ressources interactionnelles cruciales au moment du déclenchement, du développement, et éventuellement de la résolution du désaccord. Le déclenchement indique le moment où le désaccord surgit dans la conversation, par exemple dans l'extrait (2) ci-dessous où la suggestion de JUL est réfutée par LAU par l'expression du «non $\backslash »^{2}$ :

JUL ça doit avoir la taille de: ((inspiration par le nez)) Besançon ${ }^{\circ}$ je dirais ${ }^{\circ}$ LAU ouais:/ ((regard vers JUL)) non $\backslash$

(2)

résolution quant à elle arrive à la fin d'une séquence du désaccord, où les interactantee's passent à une nouvelle thématique, et le désaccord est éventuellement résolu comme dans l'exemple (3) ci-dessous, où ANT renonce à poursuivre le désaccord sur une recette de cuisine et donne la responsabilité à $\mathrm{BRU}^{3}$ :

BRU mais euh j'pense c'est la même [chose] ANT

[j'sais ] pas ouais mais i'faut ouais ça doit revenir peut-être au même en fait ben écoute ((tend la main vers BRU)) c'est- c'est toi qui euh: c'est- c'est toi qui diriges cette opération (par exemple «non non », « ah mais », « hein/»), paraverbal (intonation, rire), et non verbal (mimiques et gestes). Les indices qui sont apparus comme particulièrement pertinents ont ensuite été intégrés dans des questions qui ont été présentées aux apprenante-s sous différentes formes, tout d'abord par une étude pilote, et ensuite par l'étude principale.

37 L'étude pilote avait comme but de faire suite directement à Thomas et al. (2016) en testant de nouveau la méthode des questions posées oralement. Après le visionnement 
de chacun des deux enregistrements choisis, les participantees ont répondu individuellement à des questions, posées par l'analyste. Les questions étaient plus dirigées que dans l'étude de Thomas et al. (2016). Les résultats avaient montré que les détails interactionnels visés n'étaient pas relevés par les participant·e·s, possiblement en raison du caractère trop large et général des questions. Par la suite, dans le but de cibler plus directement les aspects interactionnels et d'éviter l'intervention directe d'une analyste, l'étude principale a été réalisée, cette fois sous format de questionnaires numériques.

Les participante's à cette dernière étude étaient des apprenant·e's avancée's suédophones du français L2 $(\mathrm{n}=6)$ et un groupe de contrôle de personnes natives de français $(n=4)$. Les apprenant·e's étaient des étudiant·e's universitaires, âgé'e·s entre 22 et 41 ans. Leur niveau de français était B1-B2, évalué par deux tests de langue ; un test de compréhension orale et un test de compréhension du vocabulaire et de la grammaire.

Chaque enregistrement était d'abord visionné en entier (39 secondes pour le premier enregistrement et 1 minute 46 pour le deuxième), suivi par des questions globales, et ensuite visionné dans des extraits plus courts suivi de questions plus détaillées et ciblées.

Les questions étaient ouvertes pour le niveau macro, par exemple « De quoi parle $X$ dans cet extrait? ", et fermées et précises pour le niveau micro (voir figures 1 et 2). Au niveau macro, le format des questions était des textes libres pour les questions globales, des questions à choix multiples, et des échelles Likert (Dörnyei 2003). Pour chaque question, les participante's devaient réagir à un certain nombre d'alternatives et avaient également la possibilité d'ajouter leurs propres réponses et commentaires comme illustré dans la figure 1.

\section{La langue parlée est :}

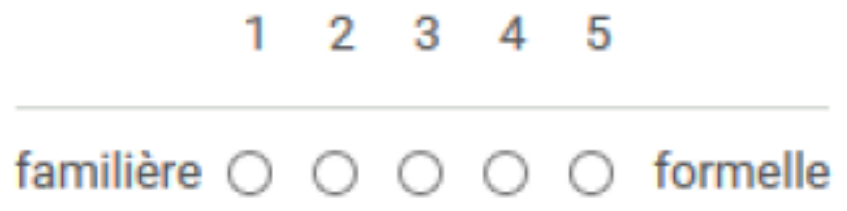

\section{Commentaires (facultatifs) :}

Figure 1 : Exemple de question ciblant le niveau macro

41 Au niveau micro les questions visaient la compréhension de différentes ressources interactionnelles qui ont été considérés comme importantes pour le déroulement du désaccord à partir de l'analyse interactionnelle. Les participant·e-s devaient interpréter des énoncés plus ou moins longs ainsi que différents gestes et mimiques. Les questions incluaient aussi différents marqueurs de désaccord et d'accord et signaux d'écoute produits par les interactante's dans les extraits, entre autres "d'accord », " oui oui », " non ", qui communiquaient différentes choses selon le contexte interactionnel (voir figure 2). 


\section{Antoine dit "ouais", "d'accord" et fait de petits hochements de tête pendant que parle Bruno. À ton avis, qu'est-ce que cela communique ? * \\ Choisir une ou plusieurs alternative(s)}

Il est d'accord avec Bruno

Il n'est pas d'accord avec Bruno

Il écoute Bruno

II n'écoute pas Bruno

Il ne communique rien

Autre (spécifier ci-dessous)

\section{Commentaires (facultatifs) :}

Figure 2 : Exemple de question ciblant le niveau micro (plusieurs choix possibles)

42 Les questions du niveau micro étaient posées après un visionnement d'extraits des deux enregistrements, pour que les participante-s ne doivent pas traiter une séquence trop longue avant de répondre aux questions. Le but était de voir si les apprenant·e's et les personnes natives comprenaient ces ressources de la même manière, ou s'il y avait des divergences.

43 Afin de pouvoir cerner l'interprétation globale de «l'atmosphère » et du désaccord reflété par le comportement des interactant·es, les participant·ess devaient également indiquer l'état d'esprit des interactant·ess sur des échelles de Likert, plus précisément à quel degré un·e interactant·e était énervée, amusée, étonné·e, mal à l'aise, ou 'autre' (choix libre pour les participantees).

\subsubsection{Résultats}

Les résultats de l'étude de Morehed (2017) ont montré que les deux groupes de participantee's avaient une compréhension très similaire du niveau macro, ce qui suggère que ce niveau ne pose pas de problèmes à des apprenantee's ayant dépassé le niveau débutant en L2. Les réponses des participant·es étaient également similaires à ce qui avait été relevé dans les analyses interactionnelles. Les apprenant·e's ont donc répondu qu'il s'agissait de deux conversations familières se déroulant dans un lieu privé, entre des interactante's ayant des relations interpersonnelles familières (contrairement à des relations professionnelles). Les apprenante's comprenaient largement ce que disaient les interactante's, et comprenaient le sujet des deux désaccords. Les résultats suivent ceux de Thomas et al. (2016) pour les deux enregistrements choisis malgré les différences de difficultés pressenties au niveau de l'analyse. 

celles des personnes natives. Ces différences s'appliquent à la compréhension d'un nombre de ressources interactionnelles dans les enregistrements, ainsi qu'à l'interprétation de la gravité du désaccord, perçu comme plus fort par les apprenant·e's que par les natife-s, où les natifees ont interprété les mots, expressions et gestes produits par les interactante's comme faisant partie d'une discussion entre amis. Les apprenante-s, de leur côté, ont interprété les mêmes ressources interactionnelles comme des vrais désaccords entre les interactante's. Cela pourrait montrer une compréhension différente $\mathrm{du}$ désaccord en interaction en français chez les apprenant·-s. Cette différence se retrouve aussi dans les réponses sur l'état d'esprit des interactant-e's dans les enregistrements. Les apprenante-s ont rapporté à un plus haut degré que le groupe de contrôle que les interactant·e's étaient mal à l'aise, énervée•ss, et étonnée'es, là où le groupe de contrôle avait tendance à indiquer que les interactant·e's étaient plutôt amusée'es.

autre résultat important est la plus grande homogénéité des réponses à l'intérieur du groupe de contrôle qu'à l'intérieur du groupe d'apprenant·es. Les apprenant·e·s ont plus souvent indiqué plusieurs choix comme réponses alors que le groupe de contrôle en a souvent choisi un seul. Une explication à ce résultat pourrait être que les apprenante-s ont plus de difficultés au niveau de la compréhension des ressources interactionnelles que le groupe de contrôle. Ces résultats signaleraient que les apprenante's ne sont pas toujours très sûrees de ce qui se passe au niveau micro de l'interaction. Mais il nous faut bien sûr plus d'études avec plus de participante-s pour pouvoir tirer des conclusions de ces résultats.

\section{Discussion méthodologique}

Comme nous l'avons montré dans cet article, l'étude du volet 'compréhension' de l'interaction ne va pas de soi et pose de nombreux défis méthodologiques. Dans le cadre d'études expérimentales basées sur des interactions authentiques, mais hors ligne, le problème méthodologique majeur est celui de faire s'exprimer les apprenante-s sur les détails interactionnels pertinents relevés dans les analyses interactionnelles, qu'ils/ elles ont peut-être de la peine à remarquer. Dans les études analysant les interactions d'apprenante's, on peut mesurer le niveau de compréhension de ce que produisent les apprenante-s, mais cela est plus difficile hors interaction. Ainsi nos études sur la compréhension soulèvent un certain nombre de questions méthodologiques.

Dans les deux études présentées (Morehed 2017; Thomas et al. 2016), les méthodes employées étaient les questions posées oralement et le questionnaire numérique. L'entretien oral a l'avantage qu'il laisse l'apprenant·e s'exprimer librement, même dans sa L1, sans qu'il/elle soit obligé'e de rédiger de longs textes. En même temps, cette méthode risque d'engendrer un certain stress en raison de la présence de l'analyste. Quant au questionnaire, l'avantage est qu'il donne à l'apprenante plus de temps pour finir la tâche, sans la présence directe de l'analyste. Par contre, le risque des tâches à l'écrit est que l'apprenante prenne trop de temps pour réfléchir à la réponse, ce qui ne permet plus d'accéder à des réponses plus spontanées et directes comme ce serait le cas dans le cadre d'une interaction.

Les deux études suggèrent que les questions posées oralement ne donnent pas de retour sur les détails interactionnels visés dans l'étude. L'étude à questionnaire 
numérique suggère par contre, que des questions plus spécifiques risquent d'influencer les réponses des participant-e's. En effet, en utilisant des questions quasi fermées avec des alternatives de réponses, on court le risque qu'elles soient trop dirigées et trop induites par l'analyse interactionnelle. Le problème avec les questions trop ouvertes, comme dans Thomas et al. (2016), est en revanche qu'elles ne permettent pas d'obtenir des informations suffisamment précises sur ce qui était réellement compris par les apprenant·es, les apprenantee's fournissant plutôt des réponses générales sur le contenu de la séquence en question.

Ainsi les méthodes de recherche utilisées par les deux études présentées ici ne nous permettent pas réellement de cerner les difficultés des apprenant-e's au niveau de la compréhension. On ne sait donc pas pourquoi les apprenante-s n'ont pas commenté les ressources interactionnelles produites par les interactant-e's natifve's et considérées comme particulièrement pertinentes du point de vue de l'analyse conversationnelle, juste après avoir reçu l'input audio et vidéo. Il est possible que ce soit en raison des questions posées, peut-être trop tendancieuses, vagues, ou larges. Il se peut aussi que les apprenant·e's ne soient pas conscientee's des différentes ressources employées par les interactante's dans les enregistrements, ou qu'ils/elles n'aient pas le vocabulaire requis. Il est également possible que la charge cognitive soit trop grande pour qu'ils/ elles soient capables de commenter l'interaction en détails après la fin de l'input, même dans leur L1.

51 Compte tenu du petit nombre de participant-e's dans l'étude avec les questionnaires numériques (Morehed 2017), ces résultats doivent être considérés avec prudence. Ils permettent néanmoins de formuler quelques recommandations en vue de futures études sur la compréhension dans le cadre de l'interaction.

La première est la nécessité de concevoir une expérience où l'input est constitué d'un matériel authentique, choisi à partir d'un corpus d'interactions authentiques. Le matériel utilisé dans les expériences devrait le plus possible refléter les spécificités d'une interaction authentique, à savoir le type d'interactions auxquelles les apprenante-s feront face en dehors de la classe de langue, par exemple dans le cadre d'une soirée entre ami-e.s. Le défi se trouve ici dans les caractéristiques mêmes du matériel authentique. N'étant pas construit dans un but didactique, chaque interaction diffère des autres quant à un grand éventail de facteurs, par exemple au niveau des relations interpersonnelles des interactantees, du sujet traité, ou du cadre spatiotemporel. Ces facteurs peuvent être contrôlés dans un matériel construit mais pas dans un matériel authentique. Par conséquent, cela requiert un choix méticuleux du matériel, ainsi que des analyses interactionnelles très fines dans le but de s'assurer que le matériel choisi reflète des situations interactionnelles similaires comparables.

53 Un autre point lié au matériel est celui du découpage des extraits choisis, à savoir la longueur. Avec un extrait plus long, par exemple toute une séquence de désaccord, le contexte interactionnel est plus large et l'apprenante aura accès à de plus amples informations sur l'interaction en question. En revanche, l'extrait risque d'être trop long et d'inclure trop d'informations pour que l'apprenante remarque et se souvienne des détails, surtout si les questions posées par la suite concernent le niveau micro. Un extrait plus court permet de viser un passage ou une ressource interactionnelle spécifique, mais aux dépens du contexte interactionnel qui par conséquent est plus restreint et limité. En raison de l'organisation séquentielle de l'interaction, où chaque énoncé est produit comme une réaction à l'énoncé précédent, en même temps qu'il va 
influencer la production du prochain, le contexte interactionnel joue un rôle important (Gülich \& Mondada 2001). Le rôle du contexte dans la compréhension dans le cadre de l'interaction est également une question importante à prendre en compte dans la réalisation des expériences.

En ce qui concerne la tâche à effectuer par les apprenant·es, il faudrait évaluer quelle serait la tâche la plus pertinente afin de cerner la partie compréhension de l'interaction chez les apprenant·e's L2. Les deux études présentées ont trouvé différents avantages et inconvénients dans le choix des méthodes. Parmi d'autres méthodes qui mériteraient d'être testées, on trouve la discussion métapragmatique à partir d'enregistrements d'interactions authentiques (Culpeper, Mackey et Taguchi 2018: 128). Il s'agit d'une discussion qui permet aux apprenante-s de s'exprimer à la fois de manière spontanée et dynamique. Il y a cependant des questions à traiter, par exemple le niveau d'intervention de l'analyste et son influence potentielle, ainsi que la constitution des groupes d'apprenantees, quant au nombre et leur niveau de la L2.

Une autre méthode possible est une tâche individuelle où l'apprenante répond oralement aux questions (cf. Bardovi-Harlig 2009). Une telle tâche pourrait comprendre une limite au niveau du temps afin d'inclure un aspect similaire à l'interaction authentique. La question à traiter concerne la modalité de retour par l'apprenante.e. Une possibilité serait que l'apprenante réponde oralement dans un microphone comme le suggère Bardovi-Harlig (2009).

\section{Conclusion}

Nous avons vu que l'étude du volet 'compréhension' de l'interaction n'est qu'indirectement traitée dans la recherche, alors que la compréhension est une partie primordiale dans l'acquisition d'une L2. Savoir interagir dans une L2 requiert plusieurs types de compétences, et constitue une partie complexe dans cette acquisition. Il faut donc en savoir plus sur la manière dont les apprenant·e's L2 gèrent et développent cette compétence.

57 Les corpus d'interactions authentiques à disposition aujourd'hui constituent une vraie ressource dans ce domaine. Ce type d'étude pose cependant de nouveaux défis méthodologiques pour la recherche. Nous avons besoin de plus d'études qui mettent à l'épreuve différentes méthodes afin d'étudier comment nous pouvons cibler précisément le volet 'compréhension' de l'interaction.

Le but à long terme est de pouvoir inclure ces aspects dans l'enseignement en L2, pour mieux préparer les apprenant·es aux interactions authentiques avec des interactant·e's natif.ve's de la L2 en question et dans le but éviter des malentendus et d'autres conséquences interactionnelles non souhaitées.

\section{Conventions de transcriptions}

59 [ chevauchement

(( production paraverbale ou non verbale

- volume bas de la voix

- amorce de mot, répétition 
/ intonation montante

: allongement vocalique

\section{BIBLIOGRAPHIE}

BARDOVI-HARLIG, K., 2009, « Conventional expressions as a pragmalinguistic resource: Recognition and production of conventional expressions in L2 pragmatics ", Language Learning 59, p. 755-795.

BARDOVI-HARLIG, K., SALSBURY, T., 2004, « The Organization of Turns in the Disagreements of L2 Learners: A Longitudinal Perspective ", dans D. Boxer \& A. D. Cohen. (eds), Studying Speaking to Inform Second Language Learning, Clevedon: Multilingual Matters, p. 199-227.

BOULTON, A., TYNE, H., 2014, Des documents authentiques aux corpus. Démarches pour l'apprentissage des langues, Paris : Didier.

BLOOMFIELD, A., WAYLAND, S C., RHOADES, E., BLODGETT, A., LINCK, J., ROSS, S., 2010, « What makes listening difficult? Factors affecting second language comprehension », College Park: University of Maryland.

CULPEPER, J., MACKEY, A., TAGUCHI, N. (eds), 2018, Second language pragmatics: from theory to research, New York: Routledge.

DEBAISIEUX, J.-M., 2009, « Des documents authentiques oraux aux corpus : un défi pour la didactique du FLE », Mélanges CRAPEL 31, p. 35-56.

DÖRNYEI, Z., 2003, Questionnaires in Second Language Research: Construction, Administration and Processing, New Jersey: Lawrence Erlbaum Associates.

GILMORE, A., 2004, « A comparison of textbook and authentic interactions », ELT Journal 58(4), p. 363-374.

GILMORE, A., 2007, « Authentic materials and authenticity in foreign language learning », Language Teaching 40, p. 97-118.

GÜLICH, E., MONDADA, L., 2001, « Analyse conversationnelle », dans G. Holtus, M. Metzeltin, C. Schmitt (eds), Lexikon der Romanistischen Linguistik (LRL), Tübingen: Max Niemeyer Verlag, p. $196-250$.

KERBRAT-ORECCHIONI, C., 1992, Les interactions verbales - Tome II, Paris : Armand Colin.

KREUTEL, K., 2007, “ “I'm not agree with you” ESL Learners' Expressions of Disagreement », TESLEJ Teaching English as a Second Foreign Language 11(3), p. 1-35.

MONDADA, L., PEKAREK DOEHLER, S., 2000, «Interaction sociale et cognition située : quels modèles pour la recherche sur l'acquisition des langues?", Acquisition et interaction en langue étrangère 12. Disponible à l'adresse : http://journals.openedition.org/aile/947.

MONDADA, L., PEKAREK DOEHLER, S., 2005, « Second Language Acquisition as Situated Practice: Task Accomplishment in the French Second Language Classroom », The Canadian Modern Language Review / La revue canadienne des langues vivantes 61(4), p. 461-490. 
MOREHED, S., 2017, Comprendre et interpréter la conversation familière en français. Une étude qualitative de six apprenant·e's avancés suédophones, Mémoire de Master, Université de Lund, sous la direction de Prof. Jonas Granfeldt, Lund (Suède). En ligne : http://lup.lub.lu.se/student-papers/ record/8899970

OURSEL, É., 2013, Des interactions de service entre francophones natifs et non natifs, Analyse de la gestion de l'intercompréhension et perspectives didactiques, thèse de doctorat, Paris : Université de la Sorbonne nouvelle - Paris III.

PEKAREK DOEHLER, S., 2012, « Conversation Analysis and Second Language Acquisition: CASLA ", dans C. Chapelle (ed.), The encyclopedia of applied linguistics. Chichester: Wiley-Blackwell, http://onlinelibrary.wiley.com/doi/10.1002/9781405198431.wbeal0217/full

POCHON-BERGER, E., PEKAREK DOEHLER, S., 2011, « Le développement de la compétence d'interaction en langue seconde : une comparaison entre deux groupes d'apprenants $\mathrm{du}$ français ", dans P. Trévisiol-okamura \& G. Komur-Thilloy (éds.), Discours, acquisition et didactique des langues, Paris : L'Harmattan, p. 243-260.

POMERANTZ, A., 1984, « Agreeing and disagreeing with assessments: some features of preferred/ dispreferred turn shapes ", dans J. M. Atkinson \& J. Heritage (eds), Structures of Social Action, Cambridge: Cambridge University Press, p. 57-101.

RAVAZZOLO, E., ETIENNE, C., 2020, « Nouvelles ressources pour le FLE à partir des études en interaction », LINX 79.

RAVAZZOLO, E., TRAVERSO, V., JOUIN-CHARDON, E., VIGNER, G., 2015, Interactions, dialogues, conversations : l'oral en français langue étrangère, Paris : Hachette.

ROST, M., 2016, Teaching and Researching - Listening, London: Longman.

SHIVELY, R. L., 2013, « Learning to be funny in Spanish during study abroad: L2 humor development », The Modern Language Journal 97(4), p. 930-946.

SHIVELY, R. L., MENKE, M. R., MANZON-OMUNDSON, S. M., 2008, « Perception of Irony by L2 Learners of Spanish », Issues in Applied Linguistics 16(2), p. 101-132.

SKOGMYR, K., PETITJEAN, C., PEKAREK DOEHLER, S., 2017, « Le développement de la compétence d'interaction en langue seconde : état des lieux et illustrations empiriques ", Revue française de linguistique appliquée 22, p. 127-145.

TAGUCHI, N., 2005, « Comprehending Implied Meaning in English as a Foreign Language », The Modern Language Journal 89, p. 543-562.

TAGUCHI, N., 2015, « Instructed pragmatics at a glance: Where instructional studies were, are, and should be going », Language Teaching 48, p. 1-50.

TAGUCHI, N., GOMEZ-LAICH, M. P., ARRUFAT-MARQUES, M.-J., 2016, « Comprehension of Indirect Meaning in Spanish as a Foreign Language », Foreign Language Annals 49, p. 677-698.

THOMAS, A., GRANFELDT, J., JOUIN-CHARDON, E., ETIENNE, C., 2016, « Conversations authentiques et CECR : compréhension globale d'interactions naturelles par des apprenant·es de FLE », Cahiers AFLS 20(1), p. 1-43.

THOMAS, J., 1983, « Cross-Cultural Pragmatic Failure », Applied Linguistics 4:2, p. 91-112.

TRAVERSO, V., 1996, La conversation familière : Analyse pragmatique des interactions, Lyon : Presses universitaire de Lyon. 
TRAVERSO, V., 2017, « Formulations, reformulations et traductions dans l'interaction : le cas de consultations médicales avec des migrants ", Revue française de linguistique appliquée 22, p. 147-164.

URSI, B., 2016, « “Et c'est qui le chef ?” Négociations et manifestations du désaccord pendant la préparation de repas ", Cahiers de praxématique 67.

\section{NOTES}

1. Corpus Clapi http://clapi.ish-lyon.cnrs.fr/FLE/affiche_contexte.php? affiche_extrait_encours=\%2089\&extrait_encours=\%2089.http://clapi.ish-lyon.cnrs.fr/FLE/ affiche_contexte.php?affiche_extrait_encours=\%2089\&extrait_encours=\%2089 2. Extrait du corpus Clapi, http://clapi.ish-lyon.cnrs.fr/V3_Feuilleter.php?num_corpus=107. 3. Extrait du corpus Clapi, http://clapi.ish-lyon.cnrs.fr/FLE/affiche_contexte.php? affiche_extrait_encours=\%2089\&extrait_encours=\%2089.

\section{RÉSUMÉS}

La mise à disposition en ligne de corpus présentant des interactions authentiques est une ressource pour l'enseignement de la compétence d'interaction qui nous permet d'illustrer les finesses spécifiques à l'interaction dans une langue donnée. Mais comment savoir ce que les apprenant·e's comprennent de ces interactions? La compréhension orale est souvent étudiée dans le cadre d'activités déconnectées de l'interaction, alors que les études qui incluent un contexte interactionnel se focalisent surtout sur la production ou font usage d'un matériel écrit et/ou construit. Pourtant dans l'objectif de former les apprenant·ess à l'interaction, une meilleure connaissance de ce qu'ils/elles comprennent ou non des interactions est nécessaire. Dans cet article nous discuterons des possibilités de tester la compréhension d'interactions authentiques par des apprenante's de FLE de différents niveaux en utilisant différentes méthodes (expérimentales) testées dans deux de nos études antérieures. Nous discuterons en particulier des protocoles utilisant la méthode des questions posées oralement (en dialogue) et celle du questionnaire numérique. L'article se terminera par une discussion des défis liés à l'étude de la compréhension dans le cadre de l'interaction, tels que la conception de la tâche, la verbalisation des réponses, la formulation des questions, et le type de matériel utilisé.

The access to online corpora presenting authentic interactions is a resource for teaching interactional competence, which allows us to illustrate the specific interactional details of a given language. However, how can we know what the L2 learners understand from these interactions? Oral comprehension is often studied through activities that are disconnected from interaction, whereas studies that include an interactional context mostly focus on production or use a written and/or invented material. However, in order to prepare the learners to interact in an L2, a better knowledge of what they do or do not understand from these is necessary. In this article, we discuss the possibilities of testing the comprehension of authentic interactions by learners of different levels of second language French, using different (experimental) methods that were tested in two of our previous studies. We discuss in particular methods with orally presented questions (in dialogue) and online questionnaires. The article concludes with a 
discussion on challenges related to the study of comprehension in an interactional context, e.g. task design, verbalization of responses, formulation of questions, and the type of material used.

INDEX

Mots-clés : compétence d'interaction, compréhension orale, FLE, matériel authentique, approche expérimentale

Keywords : interactional competence, oral comprehension, L2 French, authentic material, experimental approach

\section{AUTEURS}

\section{SIMONE MOREHED}

Doctorante, Département de plurilinguisme et didactique des langues étrangères, FLE, Université de Fribourg

\section{ANITA THOMAS}

Professeure, Département de plurilinguisme et didactique des langues étrangères, FLE, Université de Fribourg 